\title{
Development of an autophagy-related gene prognostic signature in lung adenocarcinoma and lung squamous cell carcinoma
}

\author{
Jie Zhu ${ }^{1}$, Min Wang ${ }^{2}$, Daixing Hu ${ }^{\text {Corresp. } 3}$ \\ ${ }^{1}$ Department of Intensive Care Unit, The People's Hospital of Tongliang District, Chongqing, China \\ 2 Department of Respiratory and Geriatrics, Chongqing Public Health Medical Center, Chongqing, China \\ 3 Department of Urology, The First Affiliated Hospital of Chongqing Medical University, Chongqing, China \\ Corresponding Author: Daixing Hu \\ Email address: hudaixing523@163.com
}

Purpose: There is plenty of evidence showing that autophagy plays an important role in the biological process of cancer. The purpose of this study was to establish a novel autophagy-related prognostic marker for lung adenocarcinoma (LUAD) and lung squamous cell carcinoma (LUSC).

Methods: The mRNA microarray and clinical data in The Cancer Genome Atlas (TCGA) were analyzed by using a univariate Cox proportional regression model to select candidate autophagy-related prognostic genes. Bioinformatics analysis of gene function using the Gene Ontology (GO) and the Kyoto Encyclopedia of Genes and Genomes (KEGG) platforms was performed. A multivariate Cox proportional regression model helped to develop a prognostic signature from the pool of candidate genes. On the basis of this prognostic signature, we could divide LUAD and LUSC patients into high-risk group and lowrisk groups. Further survival analysis demonstrated that high-risk patients had significantly shorter disease-free survival (DFS) than the low-risk patients. The signature which contains 6 autophagy-related genes (EIF4EBP1, TP63, BNIP3, ATIC, ERO1A and FADD) showed good performance for predicting the survival of LUAD and LUSC patients by having a better Area Under Curves (AUC) than other clinical parameters. Its efficacy was also validated by data from the Gene Expression Omnibus (GEO) database.

Conclusion: Collectively, the prognostic signature we proposed is a promising biomarker for monitoring the outcomes of LUAD and LUSC. 
1 Development of an autophagy-related gene prognostic signature in lung

2 adenocarcinoma and lung squamous cell carcinoma.

3 Jie $\mathrm{Zhu}^{1}$, Min Wang ${ }^{2}$, Daixing $\mathrm{Hu}^{3}$

4 1. Department of Intensive Care Unit, the People's Hospital of Tongliang District,

5 Chongqing, China

6 2. Department of Respiratory and Geriatrics, Chongqing Public Health Medical

7 Center, Chongqing, China

8 3. Department of Urology, the First Affiliated Hospital of Chongqing Medical

9 University, Chongqing, China

10 Corresponding Author:

11 Daixing $\mathrm{Hu}^{3}$

12 Department of Urolgy, The First Affiliated Hospital of Chongqing Medical University, No.1

13 Youyi Road, Yuan Jiagang, Yuzhong

14 District, Chongqing, 400010, China

15 Email address: hudaixing523@163.com 


\section{Development of an autophagy-related gene prognostic signature in lung adenocarcinoma} and lung squamous cell carcinoma.

\section{Abstract}

Purpose: There is plenty of evidence showing that autophagy plays an important role in the biological process of cancer. The purpose of this study was to establish a novel autophagyrelated prognostic marker for lung adenocarcinoma (LUAD) and lung squamous cell carcinoma (LUSC).

Methods: The mRNA microarray and clinical data in The Cancer Genome Atlas (TCGA) were analyzed by using a univariate Cox proportional regression model to select candidate autophagyrelated prognostic genes. Bioinformatics analysis of gene function using the Gene Ontology (GO) and the Kyoto Encyclopedia of Genes and Genomes (KEGG) platforms was performed. A multivariate Cox proportional regression model helped to develop a prognostic signature from the pool of candidate genes. On the basis of this prognostic signature, we could divide LUAD and LUSC patients into high-risk group and low-risk groups. Further survival analysis demonstrated that high-risk patients had significantly shorter disease-free survival (DFS) than the low-risk patients. The signature which contains 6 autophagy-related genes (EIF4EBP1, TP63, BNIP3, ATIC, ERO1A and FADD) showed good performance for predicting the survival of LUAD and LUSC patients by having a better Area Under Curves (AUC) than other clinical parameters. Its efficacy was also validated by data from the Gene Expression Omnibus (GEO) database.

Conclusion: Collectively, the prognostic signature we proposed is a promising biomarker for monitoring the outcomes of LUAD and LUSC. 


\section{Introduction}

Lung cancer is a fatal malignancy worldwide and is one of the leading causes of death caused by malignant tumors. In 2019, the mortality of lung cancer still ranks first among those of all kinds of cancers in the United States (Siegel et al. 2019). For worldwide, it is also the leading cause of cancer death among men and the second leading cause of cancer death among women (Torre et al. 2016). More than half (57\%) of lung cancer patients are diagnosed at the time of the distant stage (Torre et al. 2016). Even patients who underwent surgical resection, chemotherapy, radiotherapy and targeted therapy did not have significantly improved survival times. The fiveyear survival varies from $4-17 \%$, leads to a need to explore new therapeutic targets and treatments (Gray et al. 2019; Hirsch et al. 2017). According to the histological classification, lung cancer is divided into small-cell lung cancer (SCLC) and non-small-cell lung cancer (NSCLC), the latter of which accounts for approximately $85 \%$ of all cases (Neal et al. 2014). Squamous cell carcinoma and adenocarcinoma account for approximately $90 \%$ of the total NSCLC cases, which make them the most common types of lung cancer (Neal et al. 2014). The poor therapeutic effect of NSCLC is mainly due to the lack of effective indicators for detecting the development of tumors at the early stage. Therapeutic progress of NSCLC is mainly due to the advances in the molecular field and the development of new drugs that target molecular abnormalities. But the existing treatment targets are prone to inducing resistance. New treatment markers and targets are needed to achieve better prognosis. The identification of aberrant genes has been a hot topic, in which the research on autophagy has a great prospect. Autophagy is the phagocytotic process of engulfing cytoplasmic proteins, complexes or organelles. The autophagosome, a cytoplasmic double-membrane structure, can be transported into lysosome and fusion with lysosome to generate the autolysosome (Galluzzi et al. 2015; Levine \& Kroemer 2008; Maiuri et al. 2007b). The degradation products can be transported back and recycled for general cell metabolism. Generally speaking, autophagy has a dual function in the apoptosis, which means it has positive and negative effects. It is suppressed by carcinogenic proteins to 
65

66

67

prevent excessive protein degradation in stressed tumor cells. Meanwhile, persistent autophagy activation leads to apoptosis (White 2015; Ye et al. 2012). According to the present understanding, autophagy is involved in the innate and adaptive immune responses and can be induced by immune receptors such as Toll-like receptors and NLRs (nucleotide oligomerization domain-like receptors) (Cadwell 2016). It takes part in the process of antigen presentation and the development of lymphocytes (Zhong et al. 2016), which makes autophagy a possible target for improving immunotherapy in NSCLC. The relationship between autophagy and NSCLC has not been fully revealed, and some studies have suggested a role of autophagy in the targeted drug resistance. For example, in patients with NSCLC, EGFR tyrosine kinase inhibitors (EGFR-TKIs) and anaplastic lymphoma kinase inhibitors (ALK) can be used as effective treatments. EGFRTKIs can induce autophagy, and high levels of autophagy after treatment with EGFR-TKIs may also lead to autophagic death of the cells (Lee et al. 2015). Hence, the combination of EGFRTKIs with autophagy inducers may be beneficial. A similar synergistic effect can be observed with ALK inhibitor resistance (Ji et al. 2014). In this study, we revealed an autophagy-related risk signature involving six genes. This signature can be used as an independent prognostic marker for lung adenocarcinoma (LUAD) and lung squamous cell carcinoma (LUSC) patients. Our study indicates that autophagy plays an important role in LUAD and LUSC and may be a promising target for the treatment of NSCLC.

\section{Materials and methods}

\section{Autophagy-related gene datasets and Patient samples}

The gene expression datasets and clinical information of LUAD and LUSC patients were downloaded from TCGA database on September 9, 2019. The supplementary clinical information of corresponding patients was obtained from cBioPortal (www.cbioportal.org). An independent microarray NSCLC cohort was extracted from the GEO database (accession number: 
90

91

GSE3141). Overall, the expression data of 222 genes from 1,102 samples (103 normal samples and 999 tumor samples) were obtained with the TCGA dataset. A total of 111 samples from the GEO dataset GSE3141 were used in the verification group. A total of 232 genes from the HADb (Human Autophagy Database) were identified as autophagy-related genes.

\section{Procedures and Statistical analysis}

A Consensus Clustering Analysis and a Principle Components Analysis were performed by the R programming language to verify the regulatory role of autophagy in LUAD and LUSC. The R package limma was used to screen the differentially expressed autophagy-related genes. Then, we carried out a series of gene functional enrichment analyses to determine the major biological attributes, including gene GO and KEGG analyses. The GOplot package was employed to visualize the enrichment terms. A univariate Cox proportional hazard regression analysis was used to evaluate the association between overall survival (OS) or DFS and gene expression values. Next, a multivariate Cox proportional hazards regression analysis was performed using the candidate prognostic genes identified by the univariate regression analysis. The independent prognostic factors were determined by the multivariate Cox proportional hazards regression analysis, and the regression coefficient and hazard ratios (HRs) were calculated by the Cox regression model. The prediction accuracy of the risk model was determined by time-dependent Receiver Operating Characteristic (ROC) analysis. Thus, we established an autophagy-related signature that could be a prediction model in LUAD and LUSC patients. On the basis of the signature, patients were classified into high-risk and low-risk groups according to their risk score, using the median score as a cutoff point. The relationship between OS, DFS and risk grouping was verified by the Kaplan-Meier method and log-rank test using the survival and survivalROC packages. We considered a $\mathrm{P}<0.05$ significant for all comparisons.

\section{Results}


115

116

117

118

119

120

121

122

123

\section{Differentially expressed autophagy-related genes}

After extracting the expression values of 232 autophagy-related genes in LUAD and LUSC patients, we finally obtained the upregulated and downregulated genes by the Wilcoxon test. Specifically, 14 downregulated genes (DLC1, NRG3, NLRC4, DAPK2, MAP1LC3C, CCL2, HSPB8, FOS, PPP1R15A, GRID1, DRAM1, PRKCQ, DAPK1, and ITPR1) and 27 upregulated genes (ATG4D, BAK1, DDIT3, EIF4G1, IFNG, HDAC1, P4B, FADD, EGFR, VMP1, PARP1, ATC, SPHK1, BNIP3, TP73, IKBKE, PTK6, ATG9B, ERO1A, TMEM74, GAPDH, ITGB4, and EIF4EBP1) were identified. Scatter plots revealed the expression patterns of these differentially expressed genes between tumor and non-tumor tissues, as shown in Figure 1.

\section{Confirmation of autophagy-related expression patterns via Consensus Clustering Analysis and Principal Components Analysis}

By setting a $\mathrm{K}$ value of 2, we obtained the optimal CDF value and classified the patients into two clusters (Figure 2A, B, C). Principal Components Analysis showed two significantly different distribution patterns. The samples of cluster 1 and cluster 2 were distributed on the left side and the right side, respectively (Figure 2D), suggesting that autophagy may play a role in the occurrence and development of LUAD and LUSC.

\section{Functional enrichment analysis of the differentially expressed genes}

Functional enrichment analysis of the 41 differentially expressed genes offered a biological understanding of these genes. The GO term functional enrichment and the KEGG pathway enrichment analyses of these genes are summarized in Figure 3 and Figure 4.

The top enriched GO terms for biological processes were autophagy, processes utilizing autophagic mechanisms, and the intrinsic apoptotic signaling pathway. Cellular components included the autophagosome membrane, the autophagosome, and integral components of the mitochondrial outer membrane. On the basis of molecular function, genes were mostly enriched 
139

140

141

142

143

144

145

146

147

in terms of protein phosphatase binding, phosphatase binding, and p53 binding. In the KEGG pathway enrichment analysis, these genes were shown to be notably associated with pathways in the autophagy (animal), apoptosis, and bladder cancer pathways. Most of the Z-scores of enriched pathways were more than zero, indicating that most of the pathways were more likely to be enhanced.

\section{Identification of an autophagy-related risk signature for the prognosis of LUAD and LUSC}

By revealing the distinct expression patterns found in LUAD and LUSC patients, we considered that identifying an autophagy-related risk signature might be useful for predicting prognosis. A univariate Cox regression analysis was performed to establish a candidate pool of autophagyrelated genes (Figure 5A). Ultimately, five genes (HDAC1, ATG4D, TP73, EIF4EBP1 and TP63) were identified as protective factors $(\mathrm{HR}<1)$, while another five genes (BNIP3, DAPK1, ATIC, ERO1A and FADD) were identified as risk factors $(\mathrm{HR}>1)$. Subsequently, a multivariate Cox analysis was conducted. As a result, EIF4EBP1, TP63, BNIP3, ATIC, ERO1A and FADD were identified as independent prognostic indicators for DFS and selected for development of the prognostic signature.

According to the multivariate Cox proportional hazards regression model, we obtained the expression coefficient of each independent risk gene. Our prognostic model for predicting DFS based on the six genes was formed using the following formula: prognosis index $(\mathrm{PI})=(-0.170$ * expression level of EIF4EBP1) $+(-0.057 *$ expression level of TP63 $)+(0.117 *$ expression level of BNIP3 $)+(0.170 *$ expression level of ATIC $)+(0.214 *$ expression level of ERO1A $)+(0.268$ * expression level of FADD). We then calculated the risk score of each patient and used the median risk value as a cutoff point for classifying patients into a high-risk group $(\mathrm{n}=732)$ or a low-risk group $(n=370)$, as shown in Figure 5D and 5E. The heatmap of these six signaturerelated genes and the Kaplan-Meier curve depending on risk score are also displayed (Figure 5B and 5C). A significant difference in survival between the high-risk group and the low-risk group was observed. Patients in the high-risk group had a shorter OS than patients in the low-risk 
165

166

167

168

169

170

171

172

173

174

175

176

177

178

179

180

181

182

183

184

185

186

187

group (five-year survival rate $=36.7 \%$ vs. $44.9 \%, p=0.0017$ ). Similar results could also be seen with the DFS (median time $=0.344$ years vs. 0.512 years, $p<0.001$ ). The results of KaplanMeier analysis also showed a prognostic ability of each single gene. The downregulation of EIF4EBP1 was strongly correlated with inferior DFS in LUAD and LUSC patients $(\mathrm{P}<0.05$; Figure 6A). Similarly, low expression of TP63 led to inferior DFS $(\mathrm{P}<0.05$; Figure 6B). In contrast, the upregulation of ATIC, FADD and ERO1A indicated a decreased DFS $(\mathrm{P}<0.05$; Figure 5D, 5E and 5F). However, we did not observe a significant difference in DFS with regard to BNIP3 expression $(\mathrm{P}<0.05$; Figure 5C). Considering that each gene had a different value in the prognostic model, a statistical difference may not occur in each survival analysis of a single gene. ROC curves of OS and DFS were used to reveal the predictive performance of the six-gene risk signature (Figure 7). The AUC values of the signature for OS and DFS were 0.656 and 0.671 , which were obviously higher than those associated with age $(\mathrm{AUC}=0.574$ and 0.478 , respectively), sex (AUC $=0.551$ and 0.502 , respectively), tumor stage $(\mathrm{AUC}=0.634$ and 0.641 , respectively), tumor $\mathrm{T}$ stage $(\mathrm{AUC}=0.629$ and 0.648 , respectively), tumor $\mathrm{N}$ stage (AUC $=$ 0.578 and 0.633 , respectively) and tumor $\mathrm{M}$ stage ( $\mathrm{AUC}=0.501$ and 0.489 , respectively). These results indicated that the risk signature had a better ability to predict the survival of LUAD and LUSC patients than did clinical factors.

Associations between the autophagy-related risk signature and clinicopathologic features in LUAD and LUSC patients

An analysis was performed to explore the associations between clinical parameters and the risk signature (Figure 8). The results showed that the signature was significantly associated with tumor stage $(p=0.006)$, M stage $(p=0.004)$, and survival outcome $(p<0.001)$. Additionally, Student's t-test analysis also indicated that these signature-related genes were differentially expressed across various clinicopathological parameters. As shown in Figure 9, differential ATIC expression was found across different tumor stages, $M$ stages and survival outcomes. Differential expression of BNIP3 was observed across different tumor stages and M stages. EIF4EBP1 showed different expression across different sexes and survival outcomes. ERO1A, 
192

193

194

195

196

197

198

199

200

201

202

203

204

205

206

207

208

209

210

211

212

213

214

215

216

showed differential expression across sexes, tumor stages and $\mathrm{T}$ stages. A difference in the expression of FADD was observed across ages and sexes. The differential expression of TP63 was related to survival outcome, sex and M stage.

\section{The autophagy-related signature is an independent prognostic factor for LUAD and LUSC patients}

We performed a univariate Cox regression analysis and a multivariate Cox regression analysis to verify the independent predictive value of the autophagy-related signature for DFS (Figure 10). The univariate Cox analysis showed that the autophagy-related signature, tumor stage, and $\mathrm{T}$ and $\mathrm{N}$ stages were all correlated with the DFS of LUAD and LUSC patients. Then, those factors were included in a multivariate Cox analysis, which showed the autophagy-related signature to be an independent predictive factor. Thus, our results confirmed that the autophagy-related signature could be used as an independent prognostic factor in clinical practice.

\section{Validation of the autophagy-related signature via an independent cohort}

We calculated the risk score for each patient in the GEO dataset GSE3141 as an independent external validation using the same formula. The patients were divided into high-risk and lowrisk groups based on the median risk score. The Kaplan-Meier analysis confirmed the prognostic ability of our signature once again (Figure 11A). As expected, the high-risk patients had alower DFS than the low-risk patients (four-year survival rate $=25.6 \%$ vs. $52.3 \%, p=0.0079$ ). The ROCs also showed a good ability of the signature to predict survival (Figure 11B). Specifically, the AUC of our signature was 0.615 . Because of the lack of clinical data such as sex, age, and tumor stage, we could not perform ROC analysis of other clinical factors. These validation experiments confirmed the valuable ability of our risk signature to predict the prognosis of LUAD and LUSC patients. A combined application of the risk signature and other clinical features would improve our prediction of LUAD and LUSC outcomes. 


\section{DISCUSSION}

218 Autophagy is a process carried out by cells to address nutritional deficiency and other cellular

219 stresses. It is involved and regulated by a series of proteins and is closely correlated with a

220 variety of cell processes and reactions. In recent years, a large number of studies have shown that

221 autophagy is a "double-edged sword" in the occurrence and treatment of tumors. On the one

222 hand, as a survival mechanism of tumor cells facing pressure, it plays a role in protecting cells.

223 On the other hand, autophagy can result in killing tumor cells under certain conditions. Therefore,

224 autophagy is considered to be a possible regulatory point for improving the therapeutic effects of

225 tumor-targeted drugs and reducing drug resistance.

226 Autophagy has promise for improving the survival of NSCLC, but most studies usually focus on

227 the role of a particular gene related to autophagy. The large-scale databases, such as TCGA and

228 GEO, provide us with effective measures to explore gene signatures, thus providing a better

229 understanding of the relationship between autophagy and tumors. In this study, based on the

230 existing gene data of patients with NSCLC, we screened autophagy-related genes and identified

231 six key prognostic genes, all of which may be possible molecular biomarkers of prognosis and

232 potential therapeutic targets. We verified the autophagy-related genes in multiple datasets, which

233 proved that the signature had very good prognostic ability across data from multiple centers.

234 The GO and KEGG analyses were also conducted to show the molecular and biological

235 pathways enriched. The results suggested that the top enriched GO terms in terms of biological

236 processes and cellular components were highly correlated with autophagy. On the basis of

237 molecular function, p53 binding is closely related with the autophagy-related gene TP63 which

238 will be discussed in detail later.

239

240

241
In addition, in the KEGG analysis, the most significant pathway was also enriched in autophagy processes. Because of this result, we speculated that specific autophagy pattern may act as tumor promoters in the occurrence and development of NSCLC. The results of the univariate survival 
242 analysis showed that ten autophagy-related genes were associated with DFS. Further multivariate 243 survival analysis helped to identify six autophagy-related genes (EIF4EBP1, TP63, BNIP3, 244 ATIC, ERO1A and FADD) to establish a prognostic signature, which could be used as an 245 independent prognostic marker for NSCLC patients. However, the effects of autophagy are not 246 immutable; they are not the same in different kinds of tumors or at different stages. Further 247 research is still needed to explore the specific mechanism. Existing research and data reveal 248 some roles of these related genes in autophagy or tumors.

249 The protein encoded by EIF4EBP1 binds to eukaryotic translation initiation factor 4e (EIF4E) 250 and suppresses the EIF4E complex, thus affecting the mTOR (mammalian target of rapamycin) 251 signaling pathway, which has been shown to promote tumorigenesis (Karlsson et al. 2013; 252 Karlsson et al. 2011). EIF4EBP1 may promote or inhibit the development of tumors as a bi253 functional factor (Armengol et al. 2007; Cai et al. 2014; Martineau et al. 2013). In general, 254 phosphorylated EIF4EBP1 is considered to be an indicator of tumor activity, indicating a worse prognosis. Unphosphorylated EIF4EBP1 (Cai et al. 2014), on the other hand, is thought to inhibit tumor activity (Martineau et al. 2013). The TP63 gene is located on chromosome 3q27/29 and is closely related to human head, neck, esophagus, lung and skin squamous cancers (Massion et al. 2003; Senoo et al. 2001).

It encodes many subtypes of p63 transcription factors, which are members of the p53 protein family, an important hub in the transcriptional and signaling networks of the epithelial cells. Therefore, the dysregulation of TP63 is closely related to the occurrence of squamous cell carcinoma (Romano et al. 2012). The genome-wide analysis suggests that the genetic variant of TPA63 may affect susceptibility to lung adenocarcinoma (Hu et al. 2014; Hu et al. 2011; Miki et al. 2010). However, there is still a lack of research on the specific mechanism.

BNIP3 was shown to be one of the most important players involved in autophagy. It encodes proteins belonging to the Bcl-2 family, which can regulate programmed cell death under some circumstances and may impart some pro-death activity (Vande Velde et al. 2000). It is linked to 
268

269

270

271

272

273

274

275

276

277

278

279

280

281

282

283

284

285

286

287

288

289

290

291

292

autophagy through three potential mechanisms. First, it can induce mitochondrial dysfunction to activate autophagy (Scherz-Shouval \& Elazar 2011). At the same time, through competitive binding with the BCL2 complex, BNIP3 can liberate Beclin-1 to induce autophagy (Bellot et al. 2009; Maiuri et al. 2007a). In addition, by inhibiting Rheb, an upstream activator of mammalian target of rapamycin (mTOR), BNIP3 may activate autophagy by repressing mTOR (Li et al. 2007). A study on early, operable NSCLC showed that the high expression of BNIP3 was an independent predictor of poor OS (Giatromanolaki et al. 2004). In vitro experiments also confirmed that BNIP3 participated in lung cancer cell migration by interacting with aryl hydrocarbon receptor (AhR) (Tsai et al. 2017). ATIC is a protein enzyme that acts on the last two steps of the de novo purine biosynthetic pathway (Martinez-Outschoorn et al. 2017; Yamaoka et al. 1997). Little has been revealed in regard to its function in lung cancer. Recent studies have shown that inhibiting the activity of ATIC metabolites may be important for the anti-tumorigenic effects of the drug pemetrexed, which is used against NSCLC (Racanelli et al. 2009).

ERO1A is a main regulatory factor of protein disulfide isomerase (PDI), which is one of the most abundant proteins in the endoplasmic reticulum. Endoplasmic reticulum stress is reported to be associated with tumorigenesis in a variety of cancers, including NSCLC (Kim et al. 2012; Network 2013). As an important component of the endoplasmic reticulum, PDI is believed to be a marker of poor prognosis in patients with tumors, such as glioblastoma, breast cancer and hepatocellular carcinoma (Shai et al. 2003; Thongwatchara et al. 2011; Xia et al. 2017). It is not surprising that ERO1A, as the main regulator of PDI, is also associated with poor prognosis in NSCLC (Hsu et al. 2016).

FADD was originally described as an adapter molecule for apoptosis and is the key to transmitting death signals from cell surface receptors (Mouasni \& Tourneur 2018). It is closely related to autophagic cell death and tumor development. Similarly, high expression of FADD 
293

294

295

296

297

298

299

300

301

302

303

304

305

306

307

308

309

310

311

312

313

314

315

316

\section{Reference}

was observed in NSCLC, and it was considered to be associated with the increased invasive behavior of the tumor and a marker for predicting prognosis (Chen et al. 2005; Luo et al. 2018).

We summarized the relevant information of the six autophagy-related genes (Table 1). It can be seen that half of them have a two-side role in the development of cancer (in addition to BNIP3, FADD and ERO1A, a consistent tumor-promoting effect is present). These results are in agreement with the current consensus that autophagy plays a bi-functional role in tumors. As we mentioned previously, most of the current studies only target an individual gene. However, in view of the complex effect of autophagy, it may not be appropriate and beneficial to simply inhibit or induce some of the autophagy-related genes based on these findings. Our study suggests that autophagy-related genes may affect the cancer development through special pattern, and by which autophagy may show a consistent effect. Revealing these specific patterns can help the clinicians identify the high-risk types and use them as new therapeutic targets. Our signature based on autophagy-related genes also confirms this conjecture.

In summary, the molecular mechanisms play an important role in the relationship between autophagy and NSCLC. Our results are expected to be applied to clinical practice, which means it may suggest potential targeted autophagy therapies for NSCLC. Further investigations will provide more information of internal mechanisms. Our study first reveals that autophagy-related pattern may affect the prognosis of patients with LUAD and LUSC. And a signature is presented to help distinguish the high-risk patients. However, a limitation of this study is its retrospective nature. More prospective studies should be conducted to validate the prognostic function of autophagy-related signatures. We also encourage multi-center data to confirm our findings. More laboratory data based on the thought of autophagy pattern can further develop our study and provide the internal mechanisms of autophagy-related network. 
318

319

320

321

322

323

324

325

326

327

328

329

330

331

332

333

334

335

336

337

338

339

340
Armengol G, Rojo F, Castellvi J, Iglesias C, Cuatrecasas M, Pons B, Baselga J, and Ramon y Cajal S. 2007. 4E-binding protein 1: a key molecular "funnel factor" in human cancer with clinical implications. Cancer Res 67:7551-7555. 10.1158/0008-5472.CAN-07-0881

Bellot G, Garcia-Medina R, Gounon P, Chiche J, Roux D, Pouyssegur J, and Mazure NM. 2009. Hypoxia-induced autophagy is mediated through hypoxia-inducible factor induction of BNIP3 and BNIP3L via their BH3 domains. Mol Cell Biol 29:2570-2581. 10.1128/MCB.00166-09

Cadwell K. 2016. Crosstalk between autophagy and inflammatory signalling pathways: balancing defence and homeostasis. Nat Rev Immunol 16:661-675. 10.1038/nri.2016.100

Cai W, Ye Q, and She QB. 2014. Loss of 4E-BP1 function induces EMT and promotes cancer cell migration and invasion via cap-dependent translational activation of snail. Oncotarget 5:6015-6027. 10.18632/oncotarget.2109

Callegari CC, Cavalli IJ, Lima RS, Jucoski TS, Torresan C, Urban CA, Kuroda F, Anselmi KF, Cavalli LR, and Ribeiro EM. 2016. Copy number and expression analysis of FOSL1, GSTP1, NTSR1, FADD and CCND1 genes in primary breast tumors with axillary lymph node metastasis. Cancer Genet 209:331-339. 10.1016/j.cancergen.2016.06.003

Chan CY, Zhao H, Pugh RJ, Pedley AM, French J, Jones SA, Zhuang X, Jinnah H, Huang TJ, and Benkovic SJ. 2015. Purinosome formation as a function of the cell cycle. Proc Natl Acad Sci U S A 112:1368-1373. 10.1073/pnas.1423009112

Chen G, Bhojani MS, Heaford AC, Chang DC, Laxman B, Thomas DG, Griffin LB, Yu J, Coppola JM, Giordano TJ, Lin L, Adams D, Orringer MB, Ross BD, Beer DG, and Rehemtulla A. 2005. Phosphorylated FADD induces NF-kappaB, perturbs cell cycle, and is associated with poor outcome in lung adenocarcinomas. Proc Natl Acad Sci U S A 102:12507-12512. 10.1073/pnas.0500397102

Peer) reviewing PDF | (2019:10:42327:1:1:NEW 22 Nov 2019) 
341 Chien HT, Cheng SD, Chuang WY, Liao CT, Wang HM, and Huang SF. 2016. Clinical 342 Implications of FADD Gene Amplification and Protein Overexpression in Taiwanese Oral 343 Cavity Squamous Cell Carcinomas. PLoS One 11:e0164870. 10.1371/journal.pone.0164870

344 Cimino Y, Costes A, Damotte D, Validire P, Mistou S, Cagnard N, Alifano M, Regnard JF, 345 Chiocchia G, Sautes-Fridman C, and Tourneur L. 2012. FADD protein release mirrors the 346 development and aggressiveness of human non-small cell lung cancer. Br J Cancer 106:1989347 1996. 10.1038/bjc.2012.196

348 Galluzzi L, Pietrocola F, Bravo-San Pedro JM, Amaravadi RK, Baehrecke EH, Cecconi F, 349 Codogno P, Debnath J, Gewirtz DA, Karantza V, Kimmelman A, Kumar S, Levine B, Maiuri 350 MC, Martin SJ, Penninger J, Piacentini M, Rubinsztein DC, Simon HU, Simonsen A, Thorburn 351 AM, Velasco G, Ryan KM, and Kroemer G. 2015. Autophagy in malignant transformation and 352 cancer progression. EMBO J 34:856-880. 10.15252/embj.201490784

353 Giatromanolaki A, Koukourakis MI, Sowter HM, Sivridis E, Gibson S, Gatter KC, and Harris 354 AL. 2004. BNIP3 expression is linked with hypoxia-regulated protein expression and with poor 355 prognosis in non-small cell lung cancer. Clin Cancer Res 10:5566-5571. 10.1158/1078356 0432.CCR-04-0076

Gray ME, Meehan J, Sullivan P, Marland JRK, Greenhalgh SN, Gregson R, Clutton RE, Ward C, 358 Cousens C, Griffiths DJ, Murray A, and Argyle D. 2019. Ovine Pulmonary Adenocarcinoma: A 359 Unique Model to Improve Lung Cancer Research. Front Oncol 9:335. 10.3389/fonc.2019.00335

360 Greasley SE, Horton P, Ramcharan J, Beardsley GP, Benkovic SJ, and Wilson IA. 2001. Crystal 361 structure of a bifunctional transformylase and cyclohydrolase enzyme in purine biosynthesis. Nat 362 Struct Biol 8:402-406. 10.1038/87555 
365

366

367

368

369

370

371

372

373

374

375

376

377

378

379

380

381

382

383

384

385

386

387

388

$10.1016 / \mathrm{S} 0140-6736(16) 30958-8$

Hsu CH, Hsu CW, Hsueh C, Wang CL, Wu YC, Wu CC, Liu CC, Yu JS, Chang YS, and Yu CJ. 2016. Identification and Characterization of Potential Biomarkers by Quantitative Tissue Proteomics of Primary Lung Adenocarcinoma. Mol Cell Proteomics 15:2396-2410. 10.1074/mcp.M115.057026

Hu QY, Jin TB, Wang L, Zhang L, Geng T, Liang G, and Kang LL. 2014. Genetic variation in the TP63 gene is associated with lung cancer risk in the Han population. Tumour Biol 35:18631866. $10.1007 / \mathrm{s} 13277-013-1248-3$

Hu Z, Wu C, Shi Y, Guo H, Zhao X, Yin Z, Yang L, Dai J, Hu L, Tan W, Li Z, Deng Q, Wang J, Wu W, Jin G, Jiang Y, Yu D, Zhou G, Chen H, Guan P, Chen Y, Shu Y, Xu L, Liu X, Liu L, Xu P, Han B, Bai C, Zhao Y, Zhang H, Yan Y, Ma H, Chen J, Chu M, Lu F, Zhang Z, Chen F, Wang X, Jin L, Lu J, Zhou B, Lu D, Wu T, Lin D, and Shen H. 2011. A genome-wide association study identifies two new lung cancer susceptibility loci at 13q12.12 and 22q12.2 in Han Chinese. Nat Genet 43:792-796. 10.1038/ng.875

Jiang X, Tan HY, Teng S, Chan YT, Wang D, and Wang N. 2019. The Role of AMP-Activated Protein Kinase as a Potential Target of Treatment of Hepatocellular Carcinoma. Cancers (Basel) 11. $10.3390 /$ cancers 11050647

Ji C, Zhang L, Cheng Y, Patel R, Wu H, Zhang Y, Wang M, Ji S, Belani CP, Yang JM, and Ren X. 2014. Induction of autophagy contributes to crizotinib resistance in ALK-positive lung cancer. Cancer Biol Ther 15:570-577. 10.4161/cbt.28162

Karlsson E, Perez-Tenorio G, Amin R, Bostner J, Skoog L, Fornander T, Sgroi DC, Nordenskjold B, Hallbeck AL, and Stal O. 2013. The mTOR effectors 4EBP1 and S6K2 are frequently coexpressed, and associated with a poor prognosis and endocrine resistance in breast cancer: a retrospective study including patients from the randomised Stockholm tamoxifen trials. 
389

390

391

392

393

394

395

396

397

398

399

400

401

402

403

404

405

406

407

408

409

410

411

Breast Cancer Res 15:R96. 10.1186/bcr3557

Karlsson E, Waltersson MA, Bostner J, Perez-Tenorio G, Olsson B, Hallbeck AL, and Stal O. 2011. High-resolution genomic analysis of the 11q13 amplicon in breast cancers identifies synergy with 8p12 amplification, involving the mTOR targets S6K2 and 4EBP1. Genes Chromosomes Cancer 50:775-787. 10.1002/gcc.20900

Kim KM, An AR, Park HS, Jang KY, Moon WS, Kang MJ, Lee YC, Ku JH, and Chung MJ. 2018. Combined expression of protein disulfide isomerase and endoplasmic reticulum oxidoreductin 1-alpha is a poor prognostic marker for non-small cell lung cancer. Oncol Lett 16:5753-5760. 10.3892/ol.2018.9339

Kischkel FC, Hellbardt S, Behrmann I, Germer M, Pawlita M, Krammer PH, and Peter ME. 1995. Cytotoxicity-dependent APO-1 (Fas/CD95)-associated proteins form a death-inducing signaling complex (DISC) with the receptor. EMBOJ 14:5579-5588.

Kim KM, Yu TK, Chu HH, Park HS, Jang KY, Moon WS, Kang MJ, Lee DG, Kim MH, Lee JH, and Chung MJ. 2012. Expression of ER stress and autophagy-related molecules in human nonsmall cell lung cancer and premalignant lesions. Int J Cancer 131:E362-370. 10.1002/ijc.26463

Lee TG, Jeong EH, Kim SY, Kim HR, and Kim CH. 2015. The combination of irreversible EGFR TKIs and SAHA induces apoptosis and autophagy-mediated cell death to overcome acquired resistance in EGFR T790M-mutated lung cancer. Int J Cancer 136:2717-2729. $10.1002 / \mathrm{ijc} .29320$

Levine B, and Kroemer G. 2008. Autophagy in the pathogenesis of disease. Cell 132:27-42. 10.1016/j.cell.2007.12.018

Li M, Jin C, Xu M, Zhou L, Li D, and Yin Y. 2017. Bifunctional enzyme ATIC promotes propagation of hepatocellular carcinoma by regulating AMPK-mTOR-S6 K1 signaling. Cell 
412

413

414

415

416

417

418

419

420

421

422

423

424

425

426

427

428

429

430

431

432

433

434

Commun Signal 15:52. 10.1186/s12964-017-0208-8

Li Y, Wang Y, Kim E, Beemiller P, Wang CY, Swanson J, You M, and Guan KL. 2007. Bnip3 mediates the hypoxia-induced inhibition on mammalian target of rapamycin by interacting with Rheb. J Biol Chem 282:35803-35813. 10.1074/jbc.M705231200

Luo J, Chen B, Gao CX, Xie HK, Han CN, and Zhou CC. 2018. SPOP promotes FADD degradation and inhibits NF-kappaB activity in non-small cell lung cancer. Biochem Biophys Res Commun 504:289-294. 10.1016/j.bbrc.2018.08.176

Maiuri MC, Le Toumelin G, Criollo A, Rain JC, Gautier F, Juin P, Tasdemir E, Pierron G, Troulinaki K, Tavernarakis N, Hickman JA, Geneste O, and Kroemer G. 2007a. Functional and physical interaction between Bcl-X(L) and a BH3-like domain in Beclin-1. EMBO J 26:25272539. 10.1038/sj.emboj.7601689

Maiuri MC, Zalckvar E, Kimchi A, and Kroemer G. 2007b. Self-eating and self-killing: crosstalk between autophagy and apoptosis. Nat Rev Mol Cell Biol 8:741-752. 10.1038/nrm2239

Martineau Y, Azar R, Bousquet C, and Pyronnet S. 2013. Anti-oncogenic potential of the eIF4Ebinding proteins. Oncogene 32:671-677. 10.1038/onc.2012.116

Martinez-Outschoorn UE, Peiris-Pages M, Pestell RG, Sotgia F, and Lisanti MP. 2017. Cancer metabolism: a therapeutic perspective. Nat Rev Clin Oncol 14:11-31. 10.1038/nrclinonc.2016.60

Massion PP, Taflan PM, Jamshedur Rahman SM, Yildiz P, Shyr Y, Edgerton ME, Westfall MD, Roberts JR, Pietenpol JA, Carbone DP, and Gonzalez AL. 2003. Significance of p63 amplification and overexpression in lung cancer development and prognosis. Cancer Res 63:7113-7121.

Maddocks OD, and Vousden KH. 2011. Metabolic regulation by p53. J Mol Med (Berl) 89:237245. $10.1007 / \mathrm{s} 00109-011-0735-5$ 
435

436

437

438

439

440

441

442

443

444

445

446

447

448

449

450

451

452

453

454

455

456

457

Miki D, Kubo M, Takahashi A, Yoon KA, Kim J, Lee GK, Zo JI, Lee JS, Hosono N, Morizono

T, Tsunoda T, Kamatani N, Chayama K, Takahashi T, Inazawa J, Nakamura Y, and Daigo Y. 2010. Variation in TP63 is associated with lung adenocarcinoma susceptibility in Japanese and Korean populations. Nat Genet 42:893-896. 10.1038/ng.667

Mouasni S, and Tourneur L. 2018. FADD at the Crossroads between Cancer and Inflammation. Trends Immunol 39:1036-1053. 10.1016/j.it.2018.10.005

Neal RD, Hamilton W, and Rogers TK. 2014. Lung cancer. BMJ 349:g6560. 10.1136/bmj.g6560

Network TC. 2013. Corrigendum: Comprehensive genomic characterization defines human glioblastoma genes and core pathways. Nature 494:506. 10.1038/nature11903

Osborn SL, Diehl G, Han SJ, Xue L, Kurd N, Hsieh K, Cado D, Robey EA, and Winoto A. 2010. Fas-associated death domain (FADD) is a negative regulator of T-cell receptor-mediated necroptosis. Proc Natl Acad Sci U S A 107:13034-13039. 10.1073/pnas.1005997107

Prapinjumrune C, Morita K, Kuribayashi Y, Hanabata Y, Shi Q, Nakajima Y, Inazawa J, and Omura K. 2010. DNA amplification and expression of FADD in oral squamous cell carcinoma. $J$ Oral Pathol Med 39:525-532. 10.1111/j.1600-0714.2009.00847.x

Pyo JO, Jang MH, Kwon YK, Lee HJ, Jun JI, Woo HN, Cho DH, Choi B, Lee H, Kim JH, Mizushima N, Oshumi Y, and Jung YK. 2005. Essential roles of Atg5 and FADD in autophagic cell death: dissection of autophagic cell death into vacuole formation and cell death. $J$ Biol Chem 280:20722-20729. 10.1074/jbc.M413934200

Racanelli AC, Rothbart SB, Heyer CL, and Moran RG. 2009. Therapeutics by cytotoxic metabolite accumulation: pemetrexed causes ZMP accumulation, AMPK activation, and mammalian target of rapamycin inhibition. Cancer Res 69:5467-5474. 10.1158/0008-5472.CAN08-4979 
458 Romano RA, Solomon LW, and Sinha S. 2012. Tp63 in oral development, neoplasia, and 459 autoimmunity. J Dent Res 91:125-132. 10.1177/0022034511411302

460 Scherz-Shouval R, and Elazar Z. 2011. Regulation of autophagy by ROS: physiology and 461 pathology. Trends Biochem Sci 36:30-38. 10.1016/j.tibs.2010.07.007

462 Senoo M, Tsuchiya I, Matsumura Y, Mori T, Saito Y, Kato H, Okamoto T, and Habu S. 2001.

463 Transcriptional dysregulation of the p73L / p63 / p51 / p40 / KET gene in human squamous cell 464 carcinomas: expression of Delta Np73L, a novel dominant-negative isoform, and loss of 465 expression of the potential tumour suppressor p51. Br J Cancer 84:1235-1241.

466 10.1054/bjoc.2000.1735

467 Shai R, Shi T, Kremen TJ, Horvath S, Liau LM, Cloughesy TF, Mischel PS, and Nelson SF. 468 2003. Gene expression profiling identifies molecular subtypes of gliomas. Oncogene 22:4918469 4923. 10.1038/sj.onc. 1206753

470 Siegel RL, Miller KD, and Jemal A. 2019. Cancer statistics, 2019. CA Cancer J Clin 69:7-34. $471 \quad 10.3322 /$ caac. 21551

472 Spilka R, Laimer K, Bachmann F, Spizzo G, Vogetseder A, Wieser M, Muller H, Haybaeck J, 473 and Obrist P. 2012. Overexpression of eIF3a in Squamous Cell Carcinoma of the Oral Cavity 474 and Its Putative Relation to Chemotherapy Response. J Oncol 2012:901956.

$47510.1155 / 2012 / 901956$

476 Thongwatchara P, Promwikorn W, Srisomsap C, Chokchaichamnankit D, Boonyaphiphat P, and 477 Thongsuksai P. 2011. Differential protein expression in primary breast cancer and matched 478 axillary node metastasis. Oncol Rep 26:185-191. 10.3892/or.2011.1266

479 Torre LA, Siegel RL, and Jemal A. 2016. Lung Cancer Statistics. Adv Exp Med Biol 893:1-19. 480 10.1007/978-3-319-24223-1_1 
481 Tsai CH, Li CH, Cheng YW, Lee CC, Liao PL, Lin CH, Huang SH, and Kang JJ. 2017. The

482 inhibition of lung cancer cell migration by AhR-regulated autophagy. Sci Rep 7:41927.

$483 \quad 10.1038 /$ srep41927

484 Vande Velde C, Cizeau J, Dubik D, Alimonti J, Brown T, Israels S, Hakem R, and Greenberg 485 AH. 2000. BNIP3 and genetic control of necrosis-like cell death through the mitochondrial 486 permeability transition pore. Mol Cell Biol 20:5454-5468. 10.1128/mcb.20.15.5454-5468.2000

487 Wang Y, Broderick P, Matakidou A, Vijayakrishnan J, Eisen T, and Houlston RS. 2011. 488 Variation in TP63 is associated with lung adenocarcinoma in the UK population. Cancer 489 Epidemiol Biomarkers Prev 20:1453-1462. 10.1158/1055-9965.EPI-11-0042

490 White E. 2015. The role for autophagy in cancer. J Clin Invest 125:42-46. 10.1172/JCI73941

491 Xia W, Zhuang J, Wang G, Ni J, Wang J, and Ye Y. 2017. P4HB promotes HCC tumorigenesis 492 through downregulation of GRP78 and subsequent upregulation of epithelial-to-mesenchymal 493 transition. Oncotarget 8:8512-8521. 10.18632/oncotarget.14337

494 Yamaoka T, Kondo M, Honda S, Iwahana H, Moritani M, Ii S, Yoshimoto K, and Itakura M. 495 1997. Amidophosphoribosyltransferase limits the rate of cell growth-linked de novo purine 496 biosynthesis in the presence of constant capacity of salvage purine biosynthesis. J Biol Chem $497 \quad 272: 17719-17725.10 .1074 / j b c .272 .28 .17719$

498 Ye MX, Li Y, Yin H, and Zhang J. 2012. Curcumin: updated molecular mechanisms and 499 intervention targets in human lung cancer. Int J Mol Sci 13:3959-3978. 10.3390/ijms13033959

500 Zhong Z, Sanchez-Lopez E, and Karin M. 2016. Autophagy, Inflammation, and Immunity: A 501 Troika Governing Cancer and Its Treatment. Cell 166:288-298. 10.1016/j.cell.2016.05.051 


\section{Figure 1}

The differentially expressed autophagy-related genes

Figure 1. (A) The heatmaps of these 41 differently expressed autophagy-related genes. The expression level of 41 differentially expressed autophagy-related genes was displayed. The red color indicated the higher gene expression value while the green color indicated the lower gene expression value. $\mathrm{N}$ indicated non-tumor tissues. T indicated tumor tissues; (B) The Volcano plot of the differentially expressed autophagy-related genes. The red dots indicated the high expression and the green for the low expression; (C) The Boxplot of the differentially expressed autophagy-related genes. The red color indicated the higher gene expression value and the green color indicated the low gene expression vlaue. $\mathrm{N}$ indicated non-tumor tissues. T indicated tumor tissues. 
A

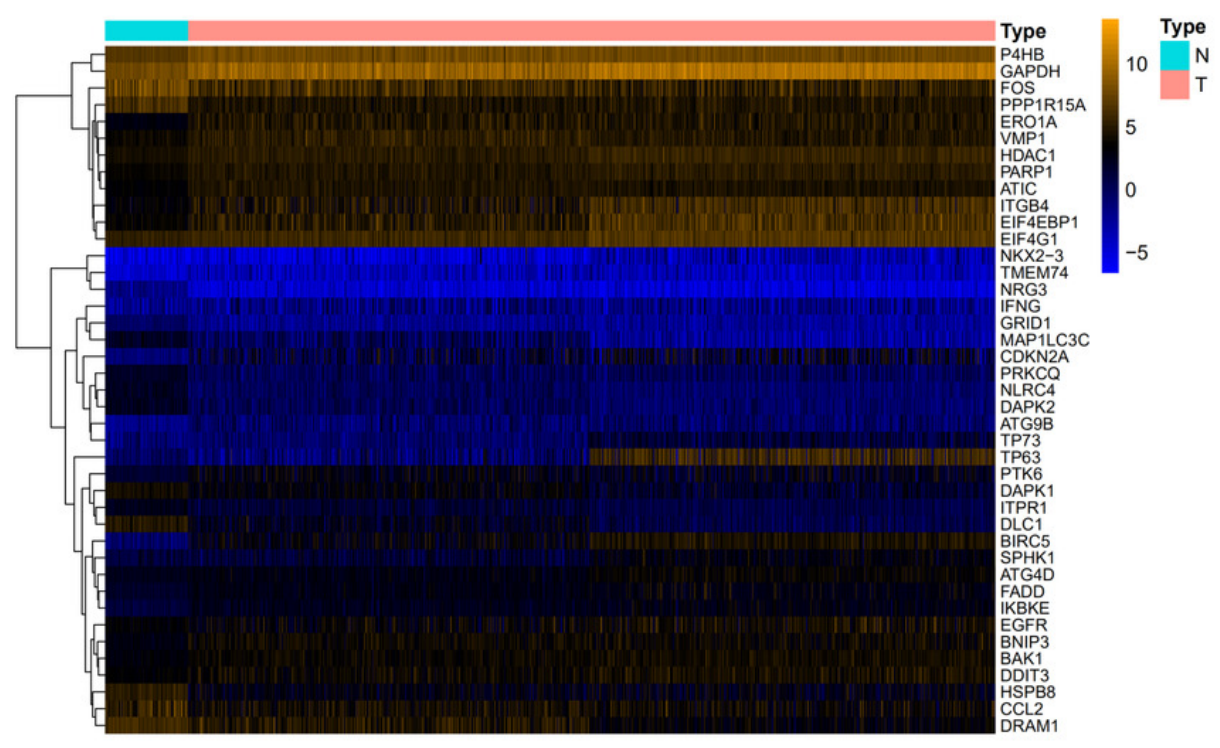

B

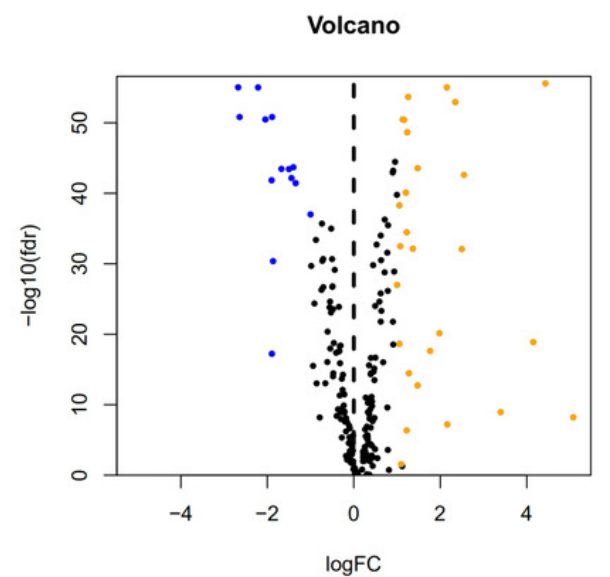

C

type 追 $\mathrm{N}$ 官 $\mathrm{T}$

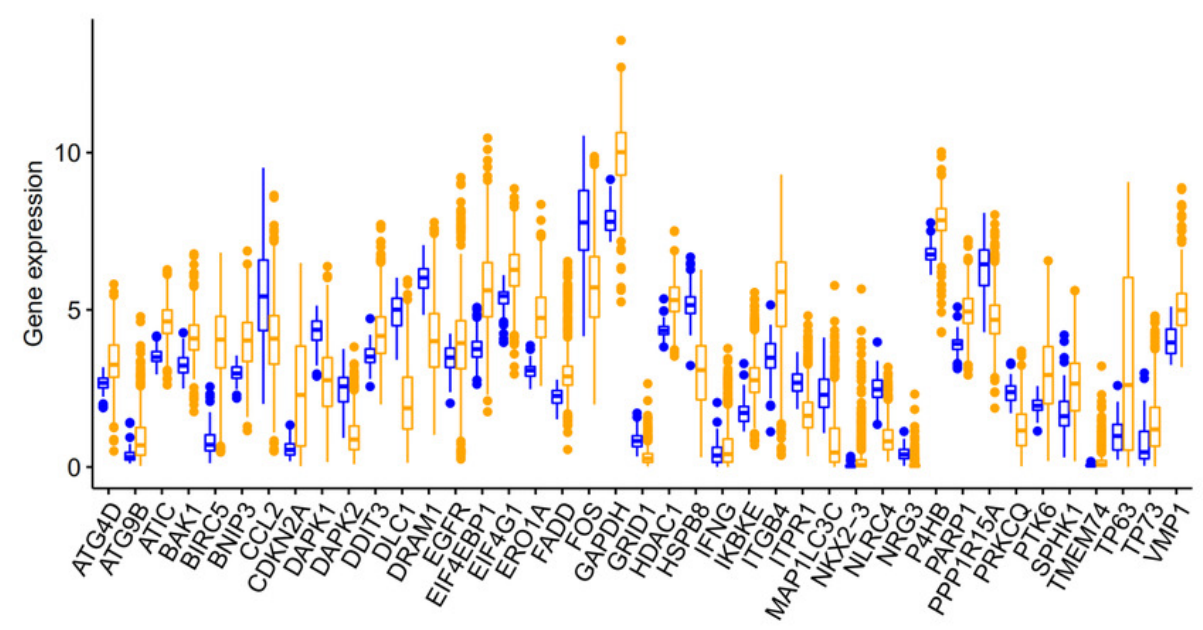


Figure 2

The Consensus Clusterin Analysis and The Principle Components Analysis

Figure 2. (A) (B) (C) The Consensus Clusterin Analysis of the autophagy-related genes, infering the optimal number of clusters, the lowest proportion of ambiguous clustering and the best CDF value by taking the K value of 2; (D) The Principle Components Analysis of the autophagy-related genes in LUAD and LUSC patients. 
A

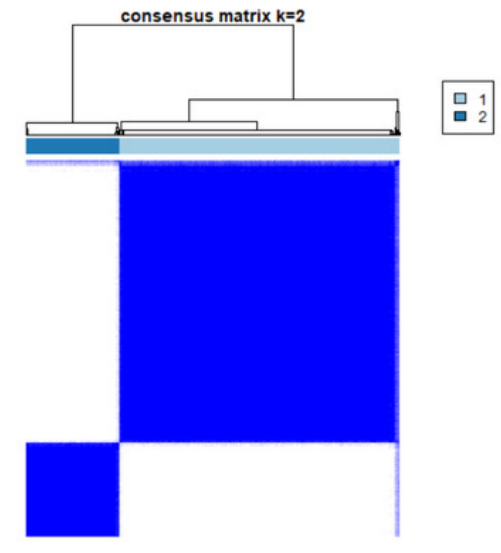

B
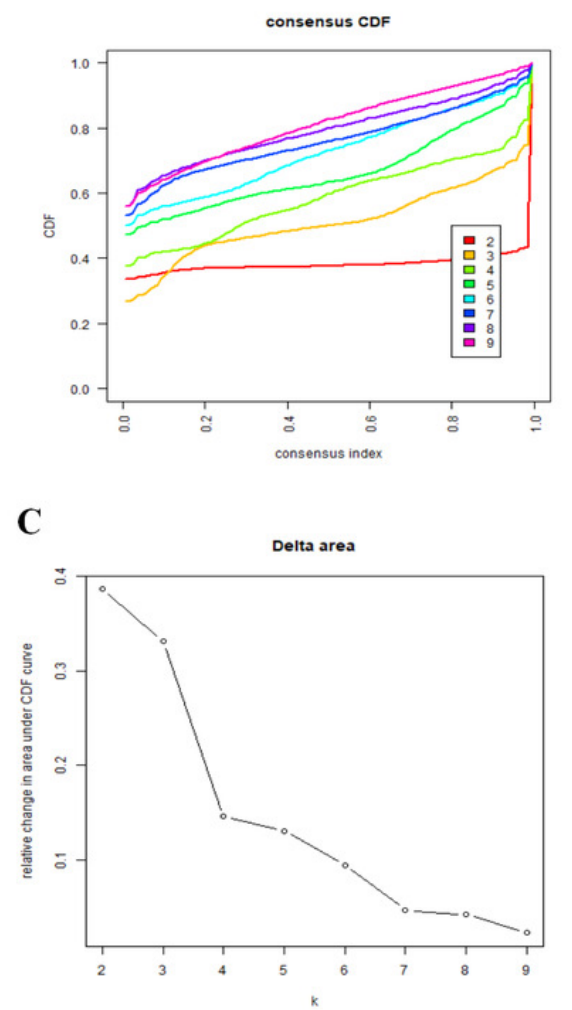

D

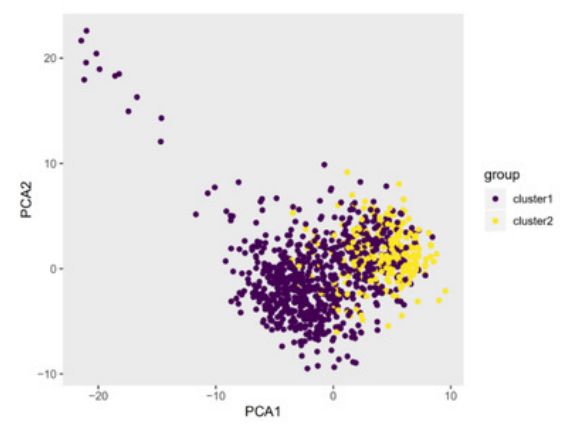

Peer) reviewing PDF | (2019:10:42327:1:1:NEW 22 Nov 2019) 


\section{Figure 3}

The Barplot and GO circle of functional enrichment analyses

Figure 3. The Barplot and GO circle of functional enrichment analyses. (A) BP indicated biological process; CC indicated cellular component; MF indicated molecular function. (B) The circle showed the scatter map of each item of the logFC of the specified gene. The red circles displayed up-regulation, and the blue ones displayed down-regulation. The higher the Z-score value indicated the higher expression of enriched pathway. 
A

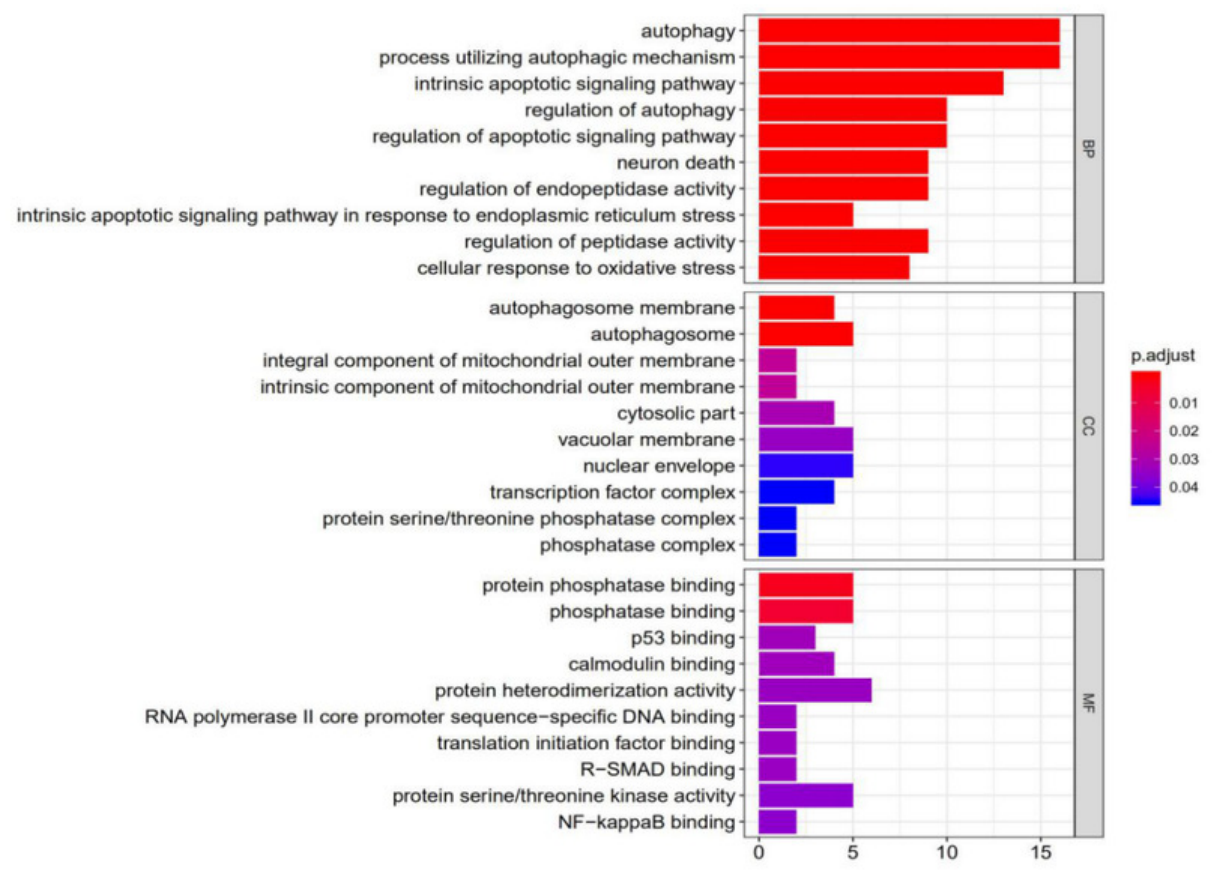

B

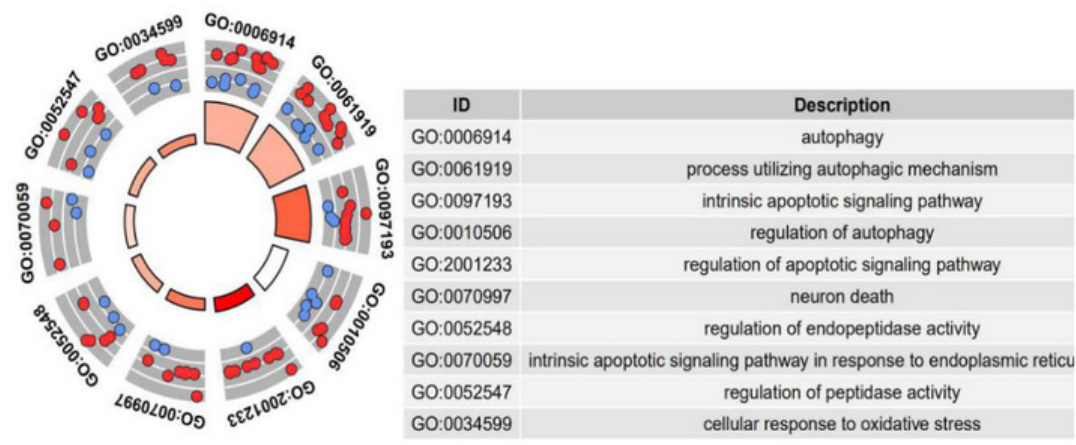

decreasing $\frac{\log \mathrm{FC}}{\text { increasing }}$ 


\section{Figure 4}

The Barplot and KEGG circle of functional enrichment analyses

Figure 4. The Barplot and KEGG circle of functional enrichment analyses. (A) The KEGG analysis of differentially expressed autophagy-related genes. (B) The circle showed the scatter map of the logFC of the specified gene. The red circles displayed up-regulation, and the blue ones displayed down-regulation. The higher the Z-score value indicated the higher expression of enriched pathway. 


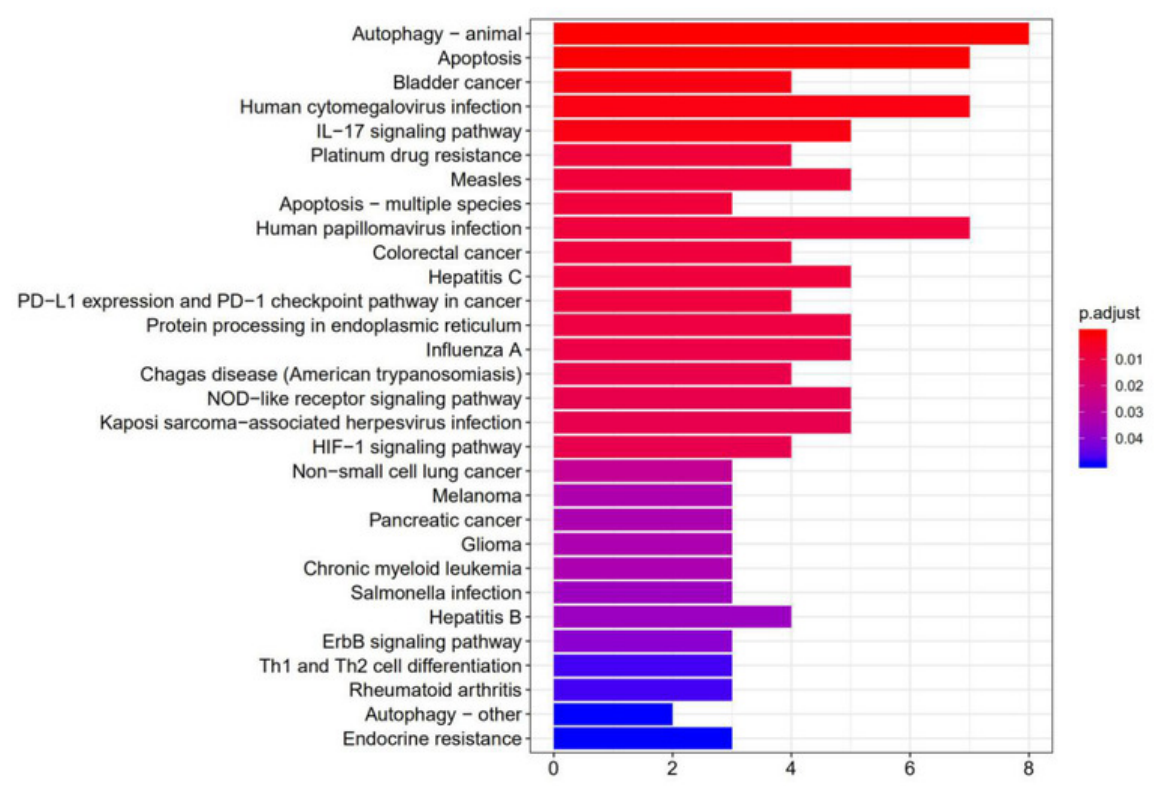

B

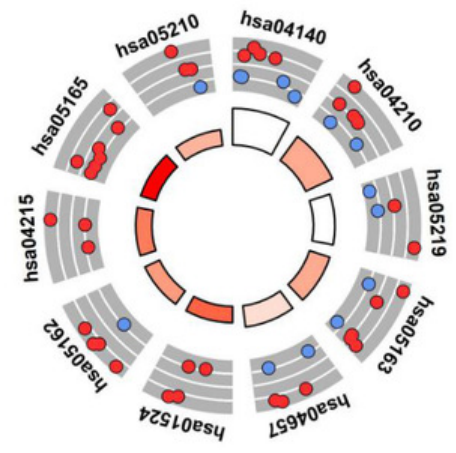

\begin{tabular}{|r|c|}
\hline \multicolumn{1}{|c|}{ ID } & $\begin{array}{c}\text { Description } \\
\text { hsa04140 }\end{array}$ \\
hsa04210 & Autophagy - animal \\
\hline hsa05219 & Apoptosis \\
\hline hsa05163 & Human cytomegalovirus infection \\
\hline hsa04657 & IL-17 signaling pathway \\
\hline hsa01524 & Platinum drug resistance \\
\hline hsa05162 & Measles \\
\hline hsa04215 & Apoptosis - multiple species \\
\hline hsa05165 & Human papillomavirus infection \\
hsa05210 & Colorectal cancer \\
\hline
\end{tabular}

\begin{tabular}{c}
$\log \mathrm{FC}$ \\
- downregulated \\
\multicolumn{2}{c}{-score } \\
decreasing increasing
\end{tabular}

z-score 


\section{Figure 5}

The autophagy-related prognostic index (PI) of LUAD and LUSC patients

Figure 5. The autophagy-related prognostic index (PI) of LUAD and LUSC patients. (A) The univariate Cox regression analysis revealed the pool of the prognosis-related genes. (B) The K-M plot represented that the high-risk group had shorter DFS than the low-risk group; (C) The heatmap of the six signature genes expression profiles; (D) The number of patients in different risk group; (E) The DFS of patients in the TCGA dataset. The red color indicated a higher risk score and the green color indicated a lower risk score. 
A

\begin{tabular}{|c|c|c|}
\hline & pvalue & Hazard ratio \\
\hline EIF4EBP1 & $<0.001$ & $0.858(0.786-0.936)$ \\
\hline ATG4D & 0.002 & $0.777(0.660-0.915)$ \\
\hline FADD & 0.006 & $1.229(1.060-1.425)$ \\
\hline ATIC & 0.044 & $1.248(1.006-1.548)$ \\
\hline HDAC1 & 0.033 & $0.802(0.655-0.982)$ \\
\hline TP63 & $<0.001$ & $0.920(0.881-0.960)$ \\
\hline BNIP3 & 0.048 & $1.149(1.001-1.318)$ \\
\hline TP73 & 0.004 & $0.829(0.729-0.943)$ \\
\hline DAPK1 & 0.042 & $1.115(1.004-1.238)$ \\
\hline ERO1A & 0.005 & $1.202(1.058-1.365)$ \\
\hline
\end{tabular}

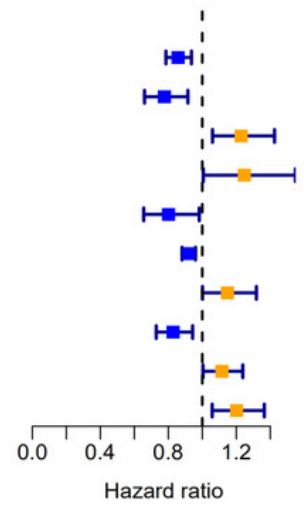

B

Risk + High risk + Low risk

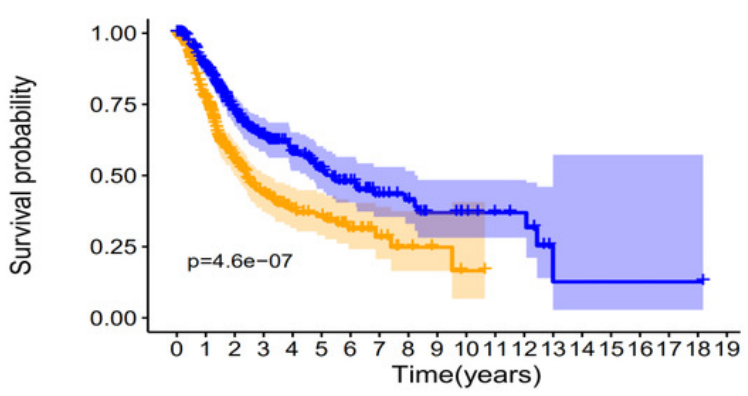

C

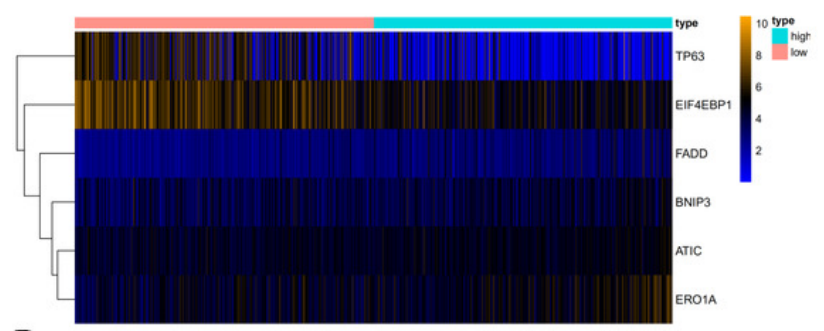

D

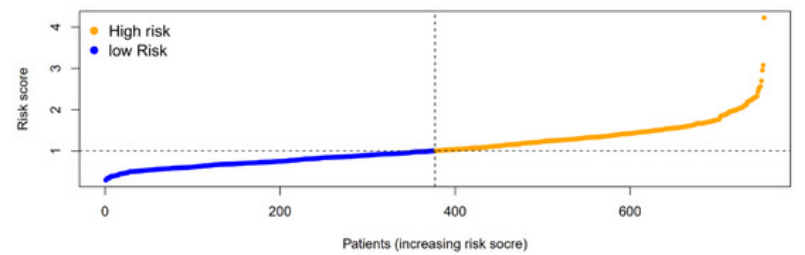

$\mathbf{E}$

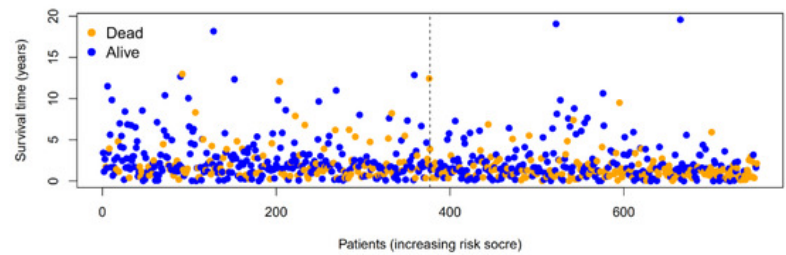




\section{Figure 6}

The correlation between six genes involved in the signature and DFS

Figure 6. The correlation between six genes involved in the signature and DFS. The K-M plots revealed (A) The expression level and DFS of EIF4EBP1, using median separation; (B) The expression level and DFS of TP63, using median separation; (C) The expression level and DFS of BNIP3, using median separation; (D) The expression level and DFS of ATIC, using median separation; (E) The expression level and DFS of ERO1A, using median separation; (F) The expression level and DFS of FADD, using median separation. 


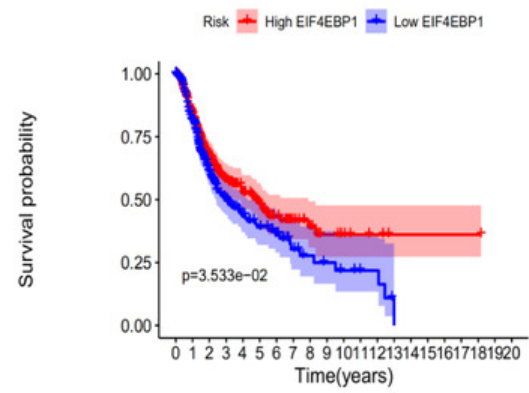

C
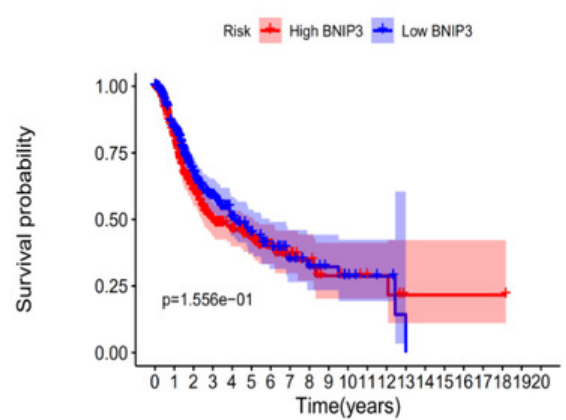

E

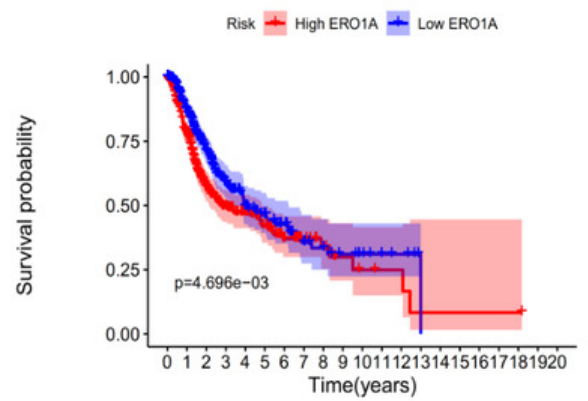

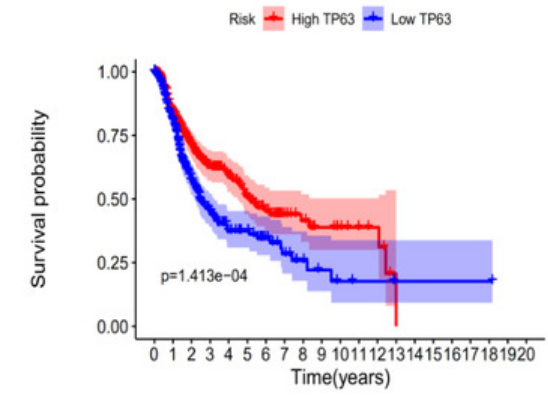

D

Risk + High ATIC + Low ATIC

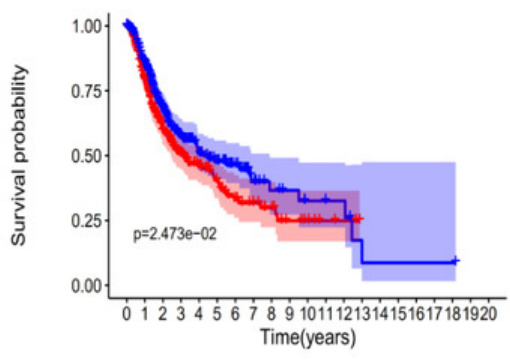

F

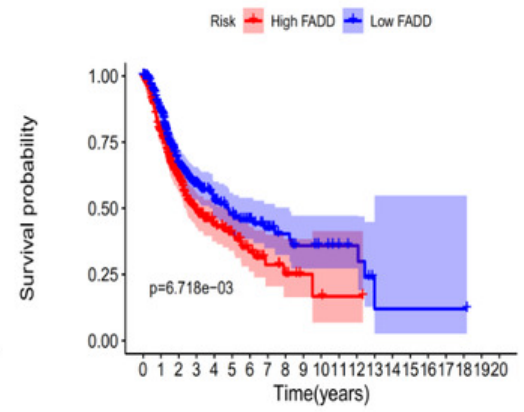


Figure 7

The ROC analysis

Figure 7. (A) The ROC analysis of OS for the signature and the clinicopathologic parameters;

(B) The ROC analysis of DFS for the signature and the clinicopathologic parameters. 
A

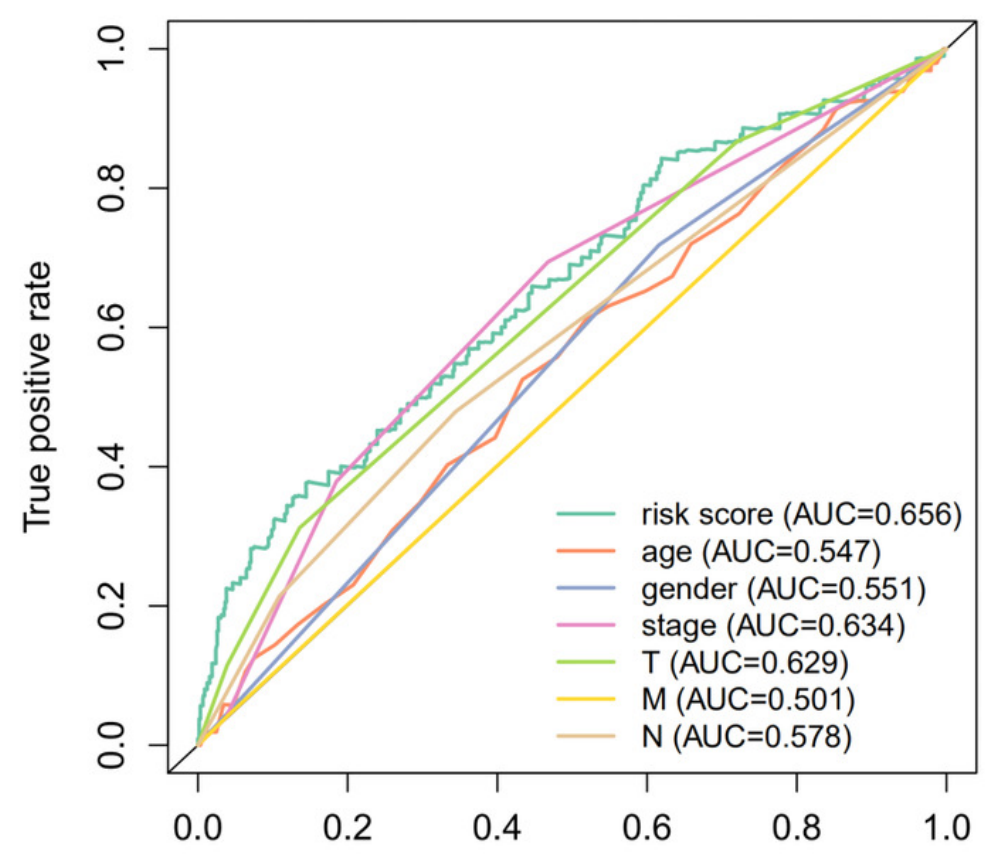

False positive rate

B

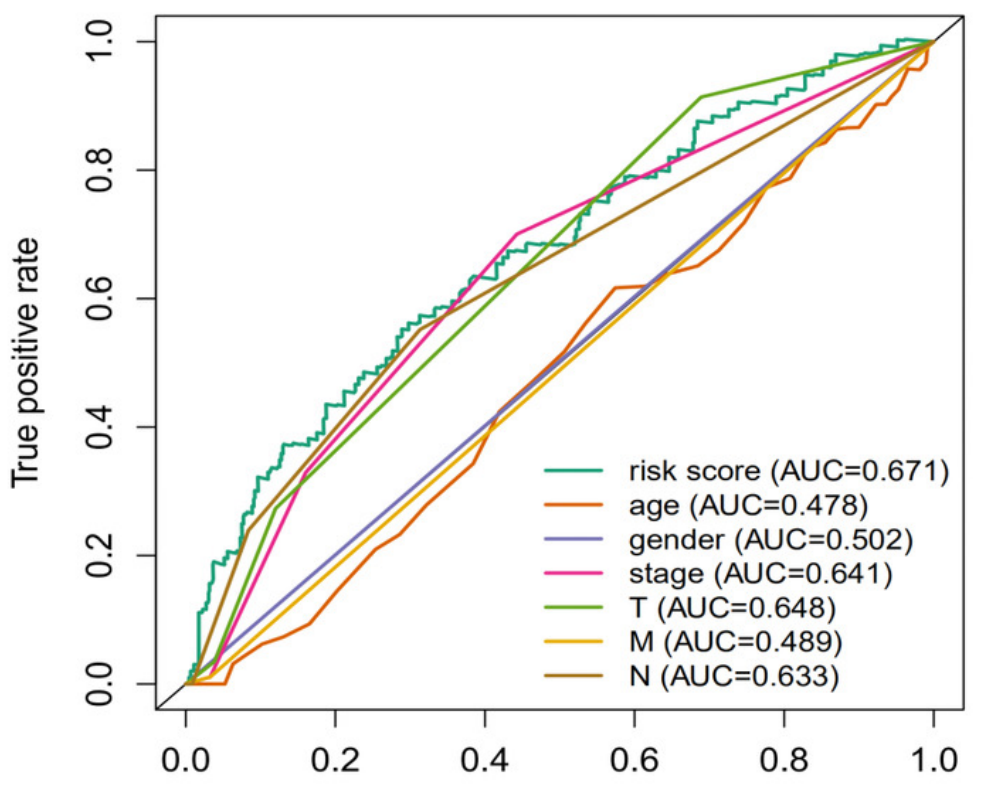

False positive rate 
Figure 8

The autophagy-related signature in the cohorts

Figure 8. The autophagy-related signature in the cohorts stratified by survival outcome ( fustat $=0$ indicated alive, fustat $=1$ indicated dead), $M$ stages $(M=0$ indicated $M 0, M=1$ indicated M1), and tumor stages (1-4). 
A

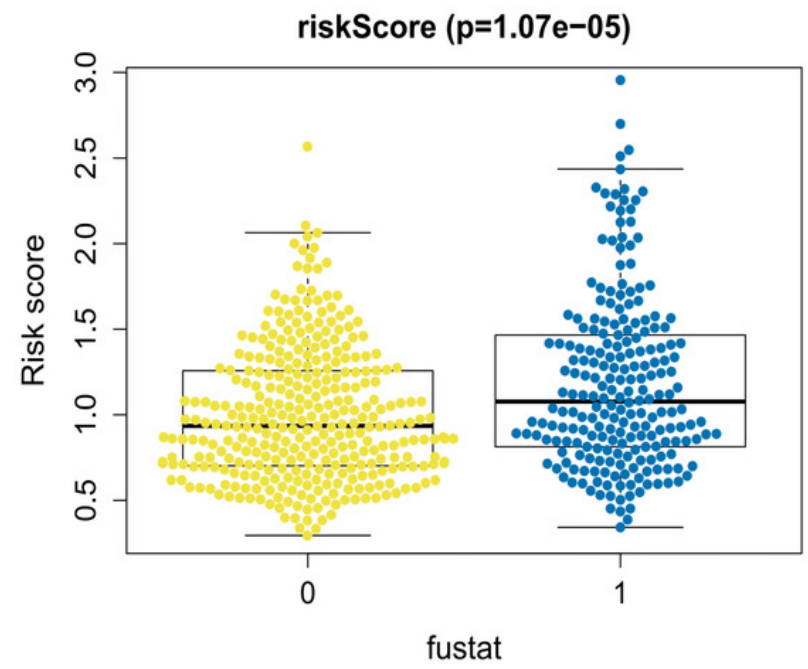

B

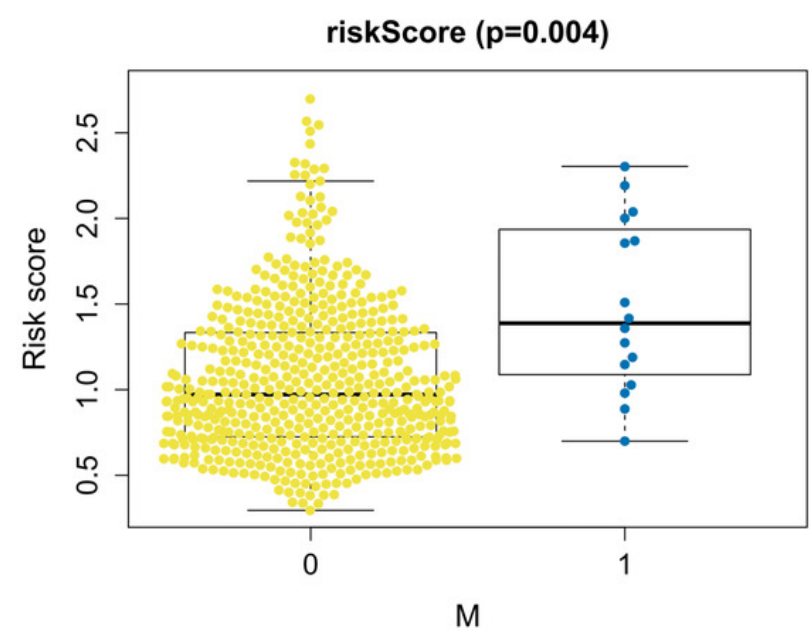

C

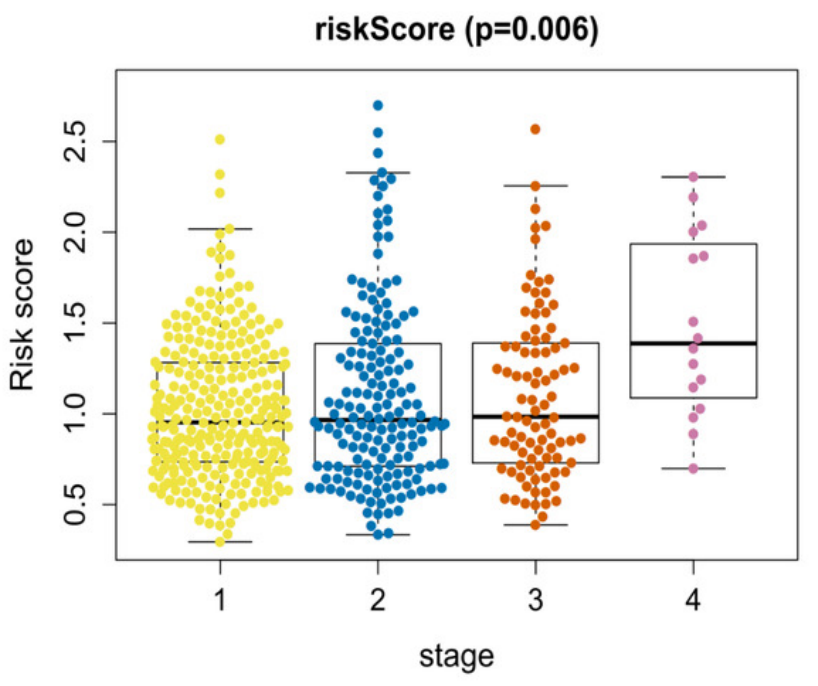


Figure 9

The signature-related genes in the cohorts

Figure 9. The signature-related genes in the cohorts stratified by survival outcome (fustat $=0$ indicated alive, fustat $=1$ indicated dead), age, gender (gender $=0$ indicated female, gender $=1$ indicated male), $T$ stages (1-4), $M$ stages ( $M=0$ indicated $M 0, M=1$ indicated $M 1$ ), and tumor stages(1-4). 


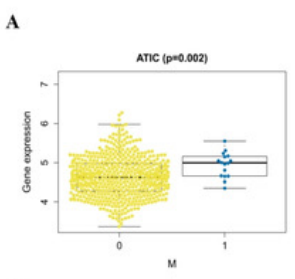

B

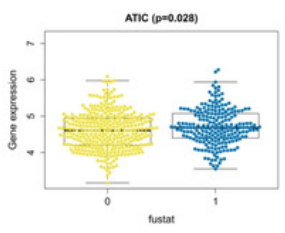

D

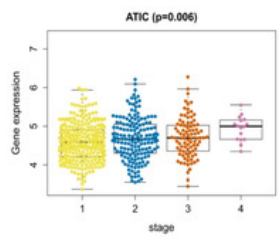

вмРP3 (p=0.007)
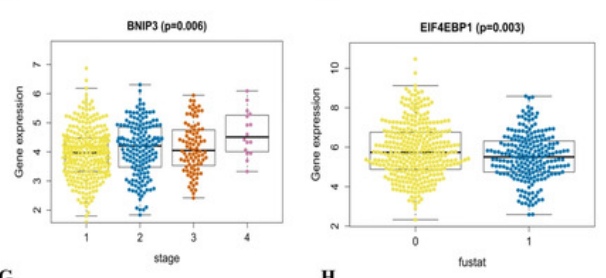

G
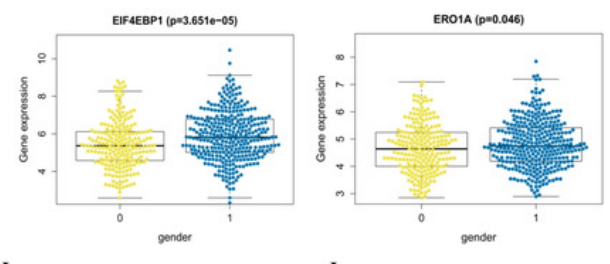
$\mathbf{J}$

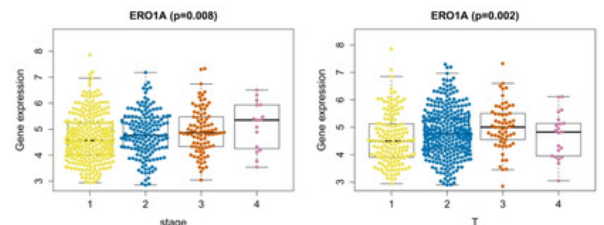

K L
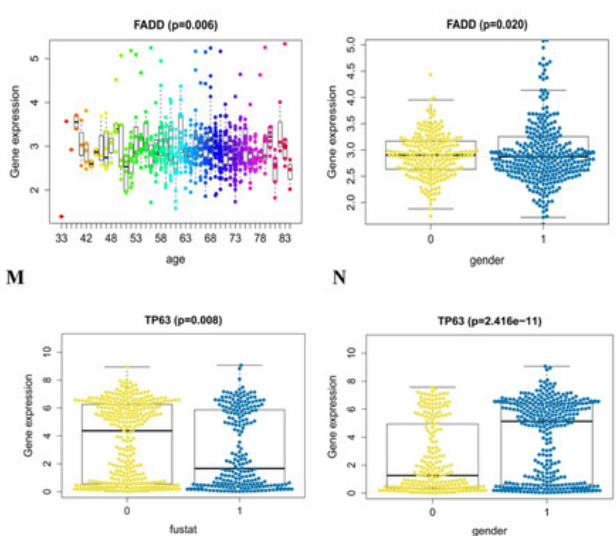

o

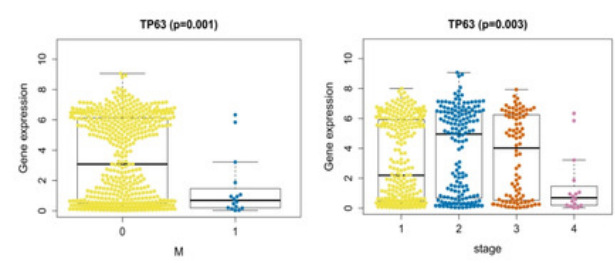




\section{Figure 10}

The univariate and multivariate Cox regression analyses

Figure 10. The univariate and multivariate Cox regression analyses verify the independent value of the autophagy-related signature.

A

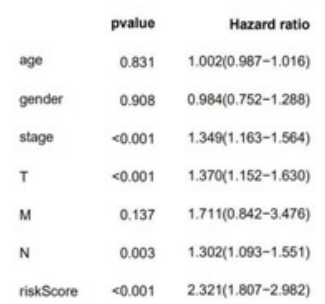

C

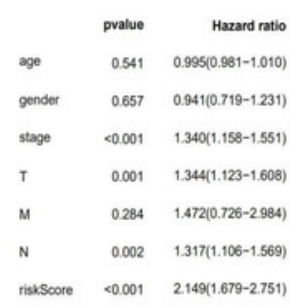

B

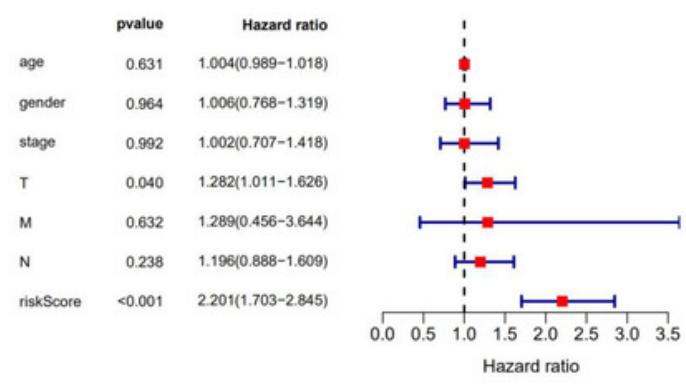

D

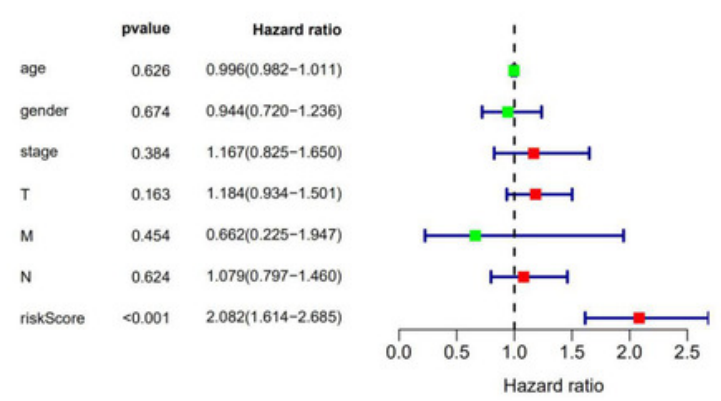


Figure 11

The K-M curves for DFS in the high-risk and the low-risk groups

Figure 11. (A) The K-M curves for DFS in the high-risk and the low-risk groups stratified by the autophagy-related signature in the GEO dataset GSE3141; (B) The ROC analysis in the GEO dataset GSE3141. 
A

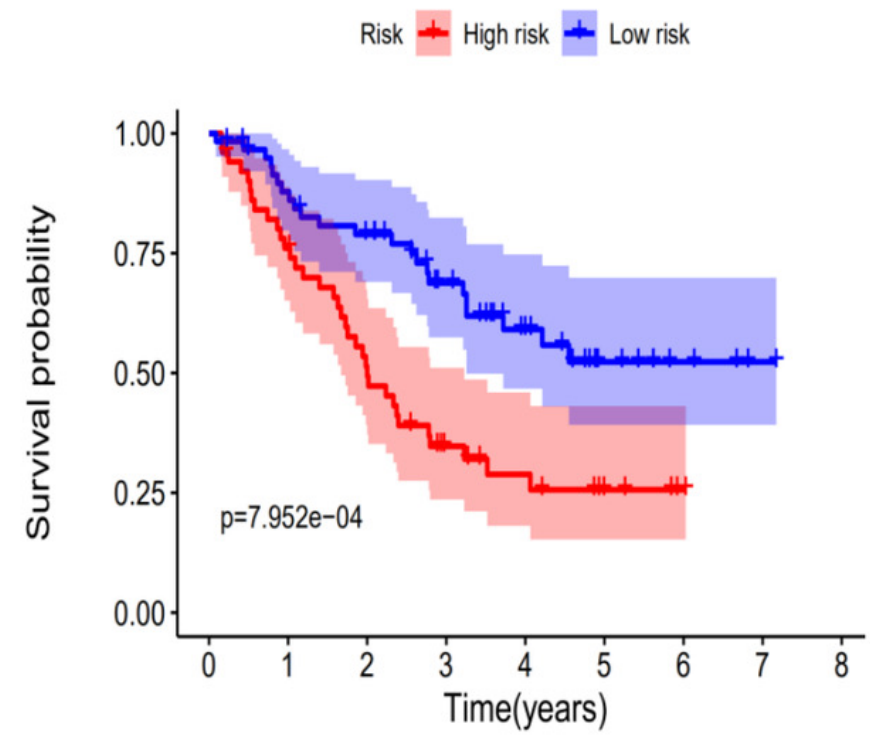

B

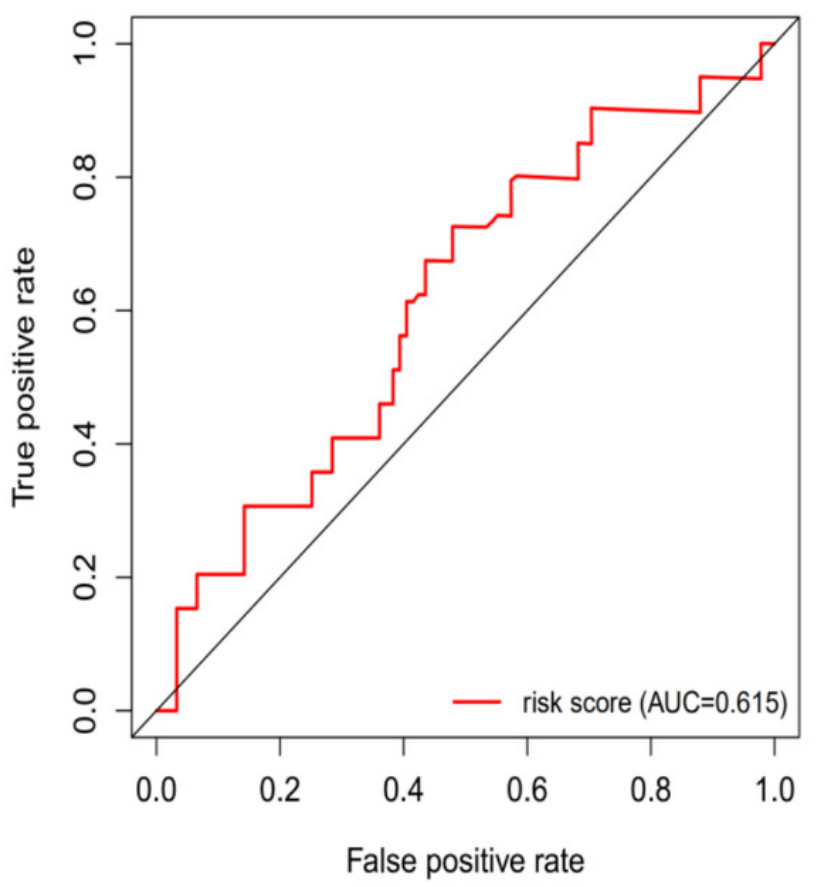




\section{Table $\mathbf{1}$ (on next page)}

Introduction and summary for the six autophagy-related genes 


\section{Table 1. Introduction and summary for the six autophagy-related genes}

\begin{tabular}{|c|c|c|c|}
\hline Gene & Functional pathway & Function & Recent Report in cancer \\
\hline EIF4EBP1 & $\begin{array}{l}\text { a translation repressor protein } \\
\text { binding to EIF4E }\end{array}$ & $\begin{array}{l}\text { inhibit EIF4E complex and the cap- } \\
\text { dependent translation to regulate mRNA } \\
\text { translation }\end{array}$ & $\begin{array}{l}\text { promote or inhibit the development of tumors as a bifunctional factor/ associate } \\
\text { with poor prognosis in breast cancer (Karlsson et al. 2011)/ act as tumor suppressor } \\
\text { in SCC (Spilka et al. 2012) }\end{array}$ \\
\hline TP63 & $\begin{array}{l}\text { the metabolic pathways, like glucose metabolism, } \\
\text { the multiple isoforms of the p63 activation of TIGAR and HK II, degradation of PGM, } \\
\text { transcription factor } \quad \text { fatty acid oxidation and mitochondrial respiration } \\
\text { (Maddocks \& Vousden 2011) }\end{array}$ & activate the autophagy gene network & $\begin{array}{l}\text { tumorigenesis and tumor suppression/ relate to the oncogenic potential role of SCC/ } \\
\text { the genetic variant rs10937405 in TP63 have been found in various ethnic } \\
\text { populations like Japanese, Korea, north Indian and British (Wang et al. } \\
\text { 2011)population and to be associated with the lung cancer risk }\end{array}$ \\
\hline BNIP3 & $\begin{array}{l}\text { a proapoptotic protein belongs to the mitochondrial dysfunction/ the production of ROS/ } \\
\text { the Bcl-2 family } \quad \text { the repression of mTOR }\end{array}$ & $\begin{array}{l}\text { regulate programmed cell death and impart } \\
\text { the pro-death activity/ induce autophagy }\end{array}$ & $\begin{array}{l}\text { a progression marker in primary human breast cancer/ be linked with poor OS in } \\
\text { NSCLC }\end{array}$ \\
\hline ATIC & $\begin{array}{l}\text { a cytosolic enzyme in the de novo the production of the intermediate FAICAR and IMP } \\
\text { purine biosynthetic pathway } \quad \text { (Chan et al. 2015; Greasley et al. 2001) }\end{array}$ & unknown & $\begin{array}{l}\text { play a significant role in the anti-tumorigenic effects in the drug of NSCLC/ be } \\
\text { related to the poor prognosis of HCC (Jiang et al. 2019; Li et al. 2017) }\end{array}$ \\
\hline ERO1A & PDI dysfunction/ unfolded protein response/ ER stress & participate in tumorigenesis & $\begin{array}{l}\text { a marker of poor prognosis in some tumors, such as glioblastoma, breast cancer and } \\
\text { hepatocellular carcinoma/ a poor prognostic factor for OS in NSCLC (Kim et al. } \\
\text { 2018) }\end{array}$ \\
\hline
\end{tabular}

a bridge between DRs and initiator pro-caspase- $8 / 10$

a key adaptor protein transmits (Kischkel et al. 1995)/ apoptosis (Mouasni \& Tourneur regulate cell cycle progression and

apoptotic signals

2018)/ interaction with ATG5 (Pyo et al. 2005)/ a proliferation

negative regulator of necroptosis (Osborn et al. 2010)

a cancer driver in oral, esophageal, laryngeal, and breast carcinomas (Callegari et al. 2016; Chien et al. 2016; Prapinjumrune et al. 2010)/ a marker for predicting prognosis in NSCLC (Cimino et al. 2012) 


\section{PeerJ}

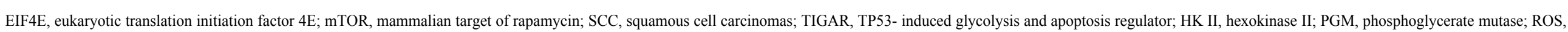

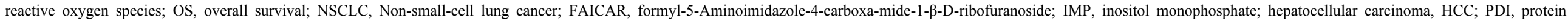
disulfide isomerase; DRs, death receptors; ATG5, autophagy-related 5. 